\title{
ASPI experiment: measurements of fields and waves on board the INTERBALL-1 spacecraft
}

\author{
S. Klimov ${ }^{1}$, S. Romanov ${ }^{1}$, E. Amata ${ }^{2}$, J. Blecki ${ }^{3}$, J. Büchner ${ }^{4}$, J. Juchniewicz ${ }^{3}$, J. Rustenbach ${ }^{4}$,P. Triska ${ }^{5}$, L. J. \\ C.Woolliscroft ${ }^{6}$, S. Savin ${ }^{1}$, Yu. Afanas'yev ${ }^{7}$, U. de Angelis $^{13}$, U. Auster ${ }^{4}$, G. Bellucci ${ }^{2}$, A. Best ${ }^{15}$, F. Farnik ${ }^{5}$, V. \\ Formisano $^{2}$, P. Gough ${ }^{8}$, R. Grard ${ }^{9}$, V. Grushin ${ }^{1}$, G. Haerendel ${ }^{4,14}$, V. Ivchenko ${ }^{16}$, V. Korepanov ${ }^{1}$, H. Lehmann ${ }^{11}$, B. \\ Nikutowski $^{4}$, M. Nozdrachev ${ }^{1}$, S. Orsini ${ }^{2}$, M. Parrot ${ }^{12}$, A. Petrukovich ${ }^{1}$, J. L. Rauch ${ }^{12}$, K.Sauer ${ }^{4}$, A. Skalsky ${ }^{1}$, J. $^{2}$ \\ Slominski ${ }^{3}$, J. G. Trotignon ${ }^{12}$, J. Vojta $^{5}$, R. Wronowski ${ }^{3}$ \\ ${ }^{1}$ Space Research Institute, Russian Academy of Sciences, Moscow, Russia \\ 2 Interplanetary Space Physics Institute, CNR, Frascati, Italy \\ ${ }^{3}$ Space Research Center, Polish Academy of Sciences, Warsaw, Poland \\ ${ }_{5}^{4}$ Max-Planck-Institut für extraterrestrische Physik, Außenstelle Berlin, Germany \\ ${ }^{5}$ Institute of atmospheric physics, Academy of Sciences of Czech Republic, Praha, Czech Republic \\ ${ }_{7}^{6}$ Department of Automatic Control and Systems Engineering, Sheffield University, Sheffield, UK \\ ${ }^{7}$ Institute of Metrology, St. Peterburg, Russia \\ ${ }^{8}$ Sussex Space Centre, Falmer, UK \\ ${ }^{9}$ Space Science Department, ESA, Noordwijk, The Netherlands \\ ${ }_{11}^{10}$ Special Design Division, Ukrainian Academy of Sciences, Lviv, Ukraine \\ ${ }^{11}$ Institute for Space Sensors, DLR, Berlin, Germany \\ ${ }^{12}$ Laboratory of Physics and Chemistry of the Environment, CNRS, Orleans, France \\ ${ }^{13}$ University of Naples, Naples, Italy \\ ${ }_{14}$ Max-Planck-Institut für extraterrestrische Physik, Garshing, Germany \\ ${ }^{15}$ Geoforschungszentrum Potsdam, Außenstelle Observatorium Niemegk, Germany \\ ${ }^{16}$ Kiev University, Kiev, Ukraine
}

Received: 14 March 1996/Revised: 14 January 1997/Accepted: 28 January 1997

\begin{abstract}
The plasma-wave experiment ASPI (analysis of spectra of plasma waves and instabilities) on board the INTERBALL spacecraft is a combined wave diagnostics experiment. It performs measurements of the DC and AC magnetic field vector by flux-gate and searchcoil sensors, the DC and AC electric field vector by Langmuir double probes and the plasma current by Langmuir split probe. Preliminary data analysis shows the low noise levels of the sensors and the compatibility of new data with the results of previous missions. During several months of in-orbit operation a rich collection of data was acquired, examples of which at the magnetopause and plasma sheet are presented in second part of the paper.
\end{abstract}

\section{Scientific objectives}

The INTERBALL project is orientated towards the investigation of the interaction between different parts of the magnetosphere in relation to changes in the solar

Correspondence to: S. Klimov wind and ionosphere. First INTERBALL-1 orbits passed through the solar wind, bow shock, magnetosheath and magnetopause regions. Several months later the orbit apogee shifted to the near-Earth magnetotail, so that INTERBALL-1 observed the tail lobes and the plasma sheet. These orbit parameters allow the neutral sheet region to be studied for several hours.

Plasma waves and fluctuations play a crucial role in the highly collisionless space plasma. Waves and fluctuations are of particular importance at the plasma boundaries such as bow shock, magnetopause, neutral sheet, and plasma sheet boundary layer. Wave-particle, interactions in the plasma result in processes like: (1) anomalous transport (pitch-angle and spatial diffusion, conductivity, viscosity); (2) energy redistribution and plasma heating; (3) generation of anisotropic distribution functions and their relaxation; (4) triggering of large-scale instabilities.

The specific objectives of the ASPI wave and field experiment on board INTERBALL-1 are:

1. The study of the fluctuation characteristics in different regions of the magnetosphere and the use of these data as high-time-resolution indicators of plasma phenomena.

2. The determination of the micro-scale plasma characteristics (e.g. diffusion and anomalous transport coefficients, wave-wave and wave-particle coupling). 
3. The study of temporal and spatial variations of macroscopic and quasi-static fields and plasma drift motions.

4. Comparative measurements with the nearby flying subsatellite and the satellite-sub-satellite combination of the INTERBALL-2.

\section{Experiment description}

In the following section and in tables all $X, Y, Z$ vector components refer to the spacecraft frame of reference (Fig. 1).

\subsection{ASPI background and overview}

The ASPI (analysis of spectra of plasma waves and instabilities) experiment is based on the method of combined wave diagnostics (CWD). This technique was used on the PROGNOZ-8,10 spacecraft (Aleksevich et al., 1983; Klimov et al., 1986), Vega-1,2 (Klimov et al., 1987), Phobos-1,2 (Grard et al., 1989) and has parallels with the S300/Geos and WEC/Cluster collaborations. The method is substantially extended in ASPI and includes:

1. The conduction of simultaneous measurements of electric field, magnetic field, plasma current, spacecraft potential and cold plasma density.

2. The use of the identical signal processing for all measured parameters.

3. The possibility to change the time-frequency resolution of measurements depending on the mode of telemetry system.

The ASPI experiment on board INTERBALL-1 is the plasma wave consortium and is composed of several instruments. A more extensive description of the experiment can be found elsewhere (Klimov et al., 1995). Locations of the ASPI sensors are in Fig. 1. Basic technical characteristics of the instruments and sensors are in Table 1. Description of the ASPI sensors and scheme of on-board processing is in the coming subsections. Analogue ASPI outputs are listed in Table 4.

Table 1. Composition of the ASPI experiment

\begin{tabular}{|c|c|}
\hline $\begin{array}{l}\text { Instrument } \\
\text { block }\end{array}$ & Description \\
\hline $\begin{array}{l}\text { MIF-M } \\
\text { BPP }\end{array}$ & $\begin{array}{l}\text { Magnetic field measurements } \\
\text { Three-component flux-gate sensor } \\
\text {-Frequency range } 0-25 \mathrm{~Hz} \\
\text {-Sensitivity } 0.005 \mathrm{nT} \text { (waveform) } \\
0.2 \mathrm{nT} \text { (DC field) }\end{array}$ \\
\hline DM2 & $\begin{array}{l}\text { Search coil sensor } \\
\text {-Frequency range } 1 \mathrm{~Hz}-40 \mathrm{kHz} \\
\text {-Sensitivity } 0.2 \mathrm{nT}(1 \mathrm{~Hz}) \\
0.004 \mathrm{nT}(10 \mathrm{~Hz}) \\
0.0004 \mathrm{nT}(100 \mathrm{~Hz})\end{array}$ \\
\hline EB-M & $\begin{array}{l}\text { Electronic box with: } \\
\text { Filter bank ( } 7 \text { filters) } \\
\text { DC/DC converter }\end{array}$ \\
\hline $\begin{array}{l}\text { PRAM } \\
\text { OPERA } \\
\text { BD1..6 }\end{array}$ & $\begin{array}{l}\text { Digital waveform processor } \\
\text { Electric field investigation } \\
\text { Three double probe sensors } \\
\text {-Frequency range } 0-150 \mathrm{kHz} \\
\text {-Sensitivity } 10^{-8} \mathrm{~V} / \mathrm{m} / \sqrt{\mathrm{Hz}}\end{array}$ \\
\hline OES & $\begin{array}{l}\text { Electronic box with: } \\
\text { Filter bank ( } 24 \text { filters) } \\
\text { DC/DC converter }\end{array}$ \\
\hline $\begin{array}{l}\text { FGM-I } \\
\text { DM1 }\end{array}$ & $\begin{array}{l}\text { Flux-gate magnetometer } \\
\text { Three-component flux-gate sensor } \\
\text {-Frequency range } 0-25 \mathrm{~Hz} \\
\text {-noise level } 0.005 \mathrm{nT} \text { at } 1 \mathrm{~Hz} \\
\text {-Dynamic range/Resolution } \\
128 \mathrm{nT} / 1.0 \mathrm{nT} \\
\text { and one-component split probe } \\
\text {-Frequency range } 0.1-40000 \mathrm{~Hz} \\
\text {-Sensitivity } 10^{-15} \mathrm{~A} / \mathrm{cm}^{2} / \sqrt{\mathrm{Hz}}\end{array}$ \\
\hline BE-DM1 & $\begin{array}{l}\text { Electronic box with: } \\
\text { analogue electronics } \\
\text { and DC/DC converter }\end{array}$ \\
\hline
\end{tabular}

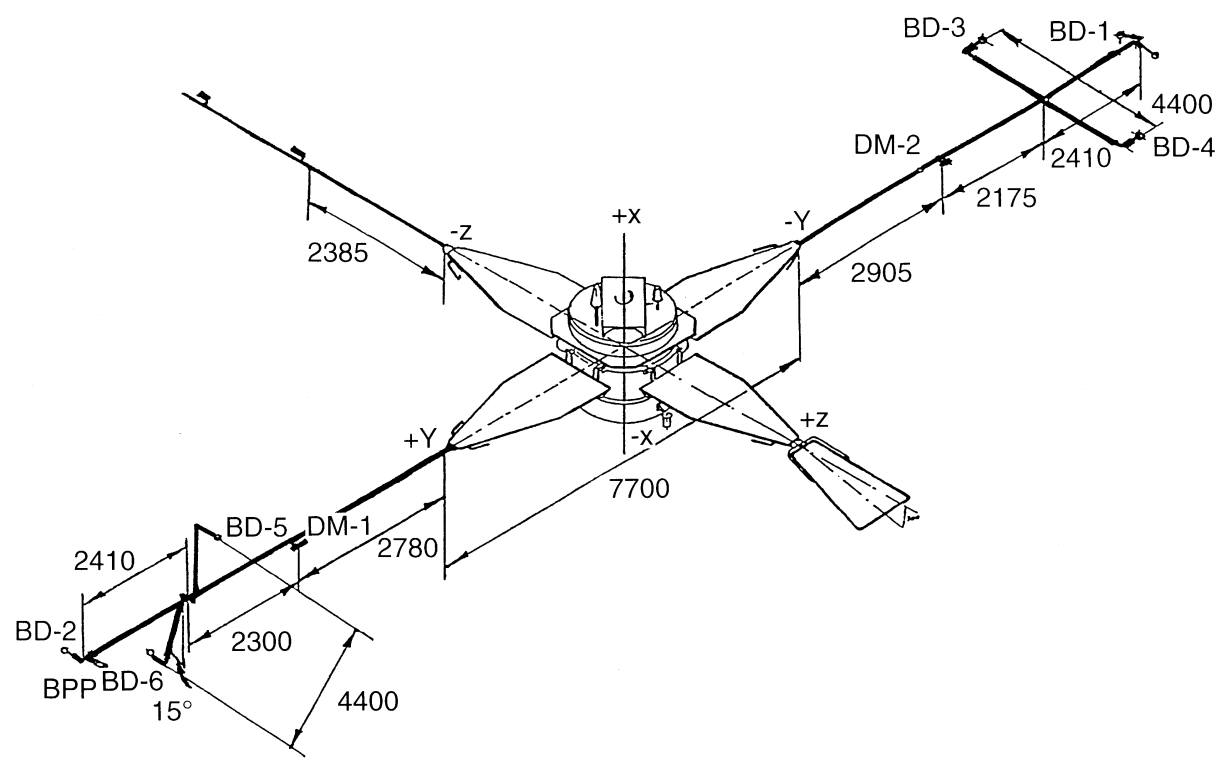

Fig. 1. Sketch of the INTERBALL-1 spacecraft: $+X$ direction is pointing to the Sun; dimensions are in $\mathrm{mm}$. ASPI sensors are shown 
Digital ASPI outputs are described in Table 2. In this section and in the tables $\bar{B}_{x, y, z}$ and $\tilde{B}_{x, y, z}$ stand for DC and AC magnetic field signals; $\bar{E}_{x, y, z}$ and $\tilde{E}_{x, y, z}$ stand for DC and AC electric field signals; $I_{z}$ stands for the plasma current signal.

ASPI instruments are now briefly described.

1. MIF-M (Russia, PI: S. Romanov). The MIF-M instrument consists of the flux-gate (BPP) and searchcoil (DM2) sensors, analogue electronics boards with lowpass and highpass filters, filter bank with seven analogue passband filters, $\mathrm{A} / \mathrm{D}$ converter and DC/DC converter.

2. OPERA (Italy, PI: E. Amata). OPERA is the electric field instrument, including six spherical Langmuir probes with the preamplifiers, mounted on the booms and the electronic subsystem with analogue, digital electronics, $\mathrm{A} / \mathrm{D}$ converter and $\mathrm{DC} / \mathrm{DC}$ converter. Analogue electronics include preamplifiers, lowpass and highpass filters and filter bank with bandpass filters. Digital electronics perform calculations of the FFT spectra, collection of housekeeping data and digital telemetry frame formatting.

3. FGM-I (Germany, PI: J. Rustenbach). The magnetometer FGM-I consists of the three-axial flux-gate sensor, the plasma current sensor and the electronic box all mounted on the boom (marked in Fig. 1 as DM1). The electronic box contains two printed boards with the analogue and drive electronics, the preamplifier of the current probe, the temperature sensor and the DC/DCconverter.

4. PRAM (Czech Rep. PI: S. Romanov). PRAM is the digital waveform processor unit. It drives the A/D converters in MIF-M, accepts digital signals from MIF$\mathrm{M}$ and processes them to fit the given bit rate. According to the mode of operation, the on-board algorithm performs the selection between amplification ranges, averaging of DC field and filter data, decimation of the $\mathrm{AC}$ data and formatting of the telemetry frames. Frames are transmitted to the digital telemetry system. Burst modes, switched on by the event selection algorithm, are possible with a buffer size equivalent to about 4 min of the prehistory information in the fastest mode. The description of the parameters processed by PRAM is in Table 2.

One more plasma current sensor is installed on board the subsatellite MAGION-4. Close collaboration with the subsatellite wave experiment is performed during periods of simultaneous measurements. This provides a good opportunity for advanced data analysis.

Table 2. ASPI parameters transmitted via PRAM in the digital form

\begin{tabular}{llll}
\hline $\begin{array}{l}\text { Physical } \\
\text { parameter }\end{array}$ & $\begin{array}{l}\text { Frequency } \\
\text { range, Hz }\end{array}$ & $\begin{array}{l}\text { Measured } \\
\text { range }\end{array}$ & $\begin{array}{l}\text { Sampling rate }(\mathrm{Hz}) \text { in } \\
\text { modes slow/normal } \\
\text { /fast/superfast }\end{array}$ \\
\hline $\bar{B}_{x, y, z}$ & $0-2$ & $0.3-300 \mathrm{nT}$ & $0.05 / 1 / 4 / 4$ \\
$\tilde{B}_{x, y, z}$ & $2-25$ & $0.005-30 \mathrm{nT}$ & $-/-/ 32 / 64$ \\
7 filters & see Table 3 & - & $0.008 / 0.0625 / 4 /-$ \\
\hline
\end{tabular}

Unfortunately, two more ASPI instruments (ADS, Poland, PI: J. Juchniewicz, J. Blecki and IFPE, Germany, PI: J. Büchner) failed to switch on, likely due to hardware problems.

\subsection{ASPI sensors}

\subsubsection{Flux-gate sensors (in MIF-M and FGM-I)}

The flux-gate sensors of the MIF-M (BPP) and of FGM-I (DM1) instruments consist of three identical flux-gate core rings, forming a right orthogonal system with fixed orientation with respect to the spacecraft body. They are mounted on the end of the boom $11 \mathrm{~m}$ (BPP) and $6.5 \mathrm{~m}$ (DM1) apart from the spacecraft spin axis (Fig. 1). They form the second, in addition to the FM3-I experiment (Nozdrachev et al., 1995), dual magnetometer system.

Signals from the BPP components are divided into two frequency ranges $0-2 \mathrm{~Hz}$ and $2-25 \mathrm{~Hz}$ (DC and AC channels). These signals are transmitted to PRAM in digital form and in the analogue form directly to spacecraft telemetry system. The signal from one of the components (selected by telecommand) is processed also by MIF-M filter bank. The signal from the DM1 sensor is transmitted in analogue form to the spacecraft telemetry system.

\subsubsection{Search-coil sensor (in MIF-M)}

The search-coil sensor of MIF-M (DM2) performs onecomponent measurements of the AC magnetic field along the spacecraft $Z$-axis (Fig. 1) in the broad frequency range (Table 1). It is mounted on the boom together with its preamplifier. The analogue signal from the sensor is transmitted to the telemetry system and processed by the filter bank in MIF-M.

\subsubsection{Langmuir split probes (in FGM-I and on the MAGION-4)}

The current sensors consist of two collectors, separated by a thin (some millimeters) insulator (Bering et al., 1973b). This method was first used in the rocket experiments (Bering et al., 1973a, b) in order to determine quasi-stationary current flows. The experiment, however, showed a high level of parasitic effects, such as photo-emission. This is not so much a problem for the AC measurements which were implemented on the PROGNOZ-10 spacecraft. The analysis of results of the PROGNOZ-10 wave experiment (Vaisberg et al., 1989; Romanov et al., 1991) has provided evidence that direct measurements of the current fluctuations in the collisionless rarefied plasma by means of split probes are possible and can be used for plasma wave diagnostics.

On board the INTERBALL-1 the current sensor is integrated in the FGM-I instrument: the cylindrical sensor itself is part of the FGM-I instrument and its 
analogue signal is transmitted to the telemetry system and to the MIF-M filter bank. On board the subsatellite, a two-component spherical current sensor is one of the inputs of the SAS instrument.

\subsubsection{Electric field sensors (in OPERA)}

The electric field experiment includes six spherical Langmuir probes with the preamplifiers (BD1-BD6), mounted on the booms (Fig. 1). The long dipole BD1BD2 $\left(E_{y}\right)$ has the length $22.68 \mathrm{~m}$. Two shorter dipoles BD3-BD4 and BD5-BD6 ( $E_{z}$ and $E_{x}$, respectively) are $4.4 \mathrm{~m}$ long. The $E_{y}$ signal input can be switched to the pairs BD3-BD5 and BD4-BD6 if necessary. The OPERA instrument can perform both potential difference and single-probe potential measurements. In the latter case probe potential is measured with respect to the spacecraft ground. All probes can be biased by the electric current in the range $\pm 100 \mu \mathrm{A}$. The bias current sweep can be performed to measure the Volt-Ampère characteristic of the probe. An estimation of the spacecraft potential is necessary for correct plasma and field measurements and can be performed using the sweep of the bias current.

Techniques of the DC and AC electric field measurements in the magnetosphere are discussed by Grard et al. (1986), Fahleson (1967), Tunally (1970) and checkedin-space experiments by Gurnett (1972), Mozer (1983), Aleksevich et al. (1983), Klimov et al. (1986, 1987), Grard et al. (1989).

The analogue electric field signal is transmitted to the telemetry system in two frequency ranges $0-3$ and $0.1-25$ $\mathrm{Hz}$ (DC and AC channels). The full frequency range signal is processed by the OPERA filter bank.

\subsection{On-board data handling}

On-board ASPI data handling algorithms include:

1. Filter-bank spectral analysis of the magnetic field and plasma current signal in MIF-M (Table 3). Inputs of the first two filters can be switched by telecommand to any magnetic field component, measured by MIF-M flux-gate sensor. Inputs of the remaining five filters can be switched between the signal from the search-coil sensor in MIF-M and the signal from the plasma current sensor in FGM-I.

Table 3. Filters in MIF-M

\begin{tabular}{lc}
\hline Sensor & Frequency range \\
& $\mathrm{Hz}$ \\
\hline BPP & $1 \ldots \ldots .4$ \\
BPP & $20 \ldots \ldots .30$ \\
DM2/DM1 & $20 \ldots \ldots .30$ \\
DM2/DM1 & $120 \ldots .150$ \\
DM2/DM1 & $350 \ldots .450$ \\
DM2/DM1 & $600 \ldots . .850$ \\
DM2/DM1 & $1900 \ldots .2100$ \\
\hline
\end{tabular}

2. FFT $(1-32 \mathrm{~Hz})$ and filter bank $(0.005-150 \mathrm{kHz})$ spectral analysis of the electric field signal in OPERA. This information is available for some limited periods.

3. All analogue ASPI outputs are transmitted to the spacecraft telemetry system (Table 4). Then they are digitized at a bit rate, determined by the telemetry operational mode. The real-time transmission mode provides a total bit rate of all ASPI analogue data equal to $16 \mathrm{kbit} / \mathrm{s}$. The duration of the real-time transmission is $2-3 \mathrm{~h}$ every $2-4$ days. Analogue data can also be recorded on the on-board tape-recorder at a low sampling rate (10-15 Mbit are available). As all ASPI analogue signals are digitized by one $\mathrm{A} / \mathrm{D}$ converter, inter-comparison of the waveform data from different ASPI instruments can be easily performed.

4. Digital telemetry frames formatted by PRAM are transmitted to the telemetry system. These frames include DC and AC magnetic field waveforms and filter-bank spectra measured by MIF-M (Table 2). OPERA housekeeping data and electric field spectra are also formatted in the digital telemetry frames and transmitted to the telemetry system. About $200 \mathrm{Mbit}$ of the on-board memory are available for the digital ASPI data between memory dumps (once every 2-4 days).

5. Waveform signals from both INTERBALL-1 and MAGION-4 current sensors can be transmitted also to the modulators of the spacecraft radio transmitters. That permits the performance of the dual-point highresolution measurements up to a frequency of about 1 $\mathrm{kHz}$. During this mode of operation, signals from both transmitters are accepted by one tracking station.

\subsection{Ground data handling}

Ground handling of ASPI data includes several stages:

1. Preparation of the quick-look data with the delay 12 days for the purpose of experiment control.

2. Preparation of the key physical parameters. The following values measured by ASPI are included in the list of parameters of the INTERBALL project: vector and total value of DC magnetic field in GSE and GSM frames of reference and amplitudes of magnetic field fluctuations in the frequency ranges $1-4$ and $600-800 \mathrm{~Hz}$.

Table 4. ASPI parameters transmitted to the spacecraft telemetry system in the analogue form

\begin{tabular}{llllc}
\hline Device/sensor & $\begin{array}{l}\text { Physical } \\
\text { parameter }\end{array}$ & $\begin{array}{l}\text { Frequency } \\
\text { range, Hz }\end{array}$ & $\begin{array}{l}\text { Measured } \\
\text { range }\end{array}$ & $\begin{array}{l}\text { Sampling } \\
\text { rate, Hz }\end{array}$ \\
\hline MIF-M/BPP & $\bar{B}_{x, y, z}$ & $0-2$ & $0.3-37.5 \mathrm{nT}$ & 8 \\
& $\tilde{B}_{x, y, z}$ & $2-25$ & $0.005-30 \mathrm{nT}$ & 64 \\
MIF-M/DM2 & $\tilde{B}_{z}$ & $0.1-500$ & $4 \cdot 10^{-6}-1$ & 256 \\
& & & $\mathrm{nT} / \sqrt{\mathrm{Hz}}$ & \\
OPERA/BD1-6 6 & $\bar{E}_{x, y, z}$ & $0-3$ & $1-570 \mathrm{mV} / \mathrm{m}$ & $8-16$ \\
& $\tilde{E}_{x, y, z}$ & $0.1-25$ & $0.9-115 \mathrm{~m} \mathrm{~V} / \mathrm{m}$ & $64-128$ \\
FGM-I/DM1 & $\bar{B}_{x, y, z}$ & $0-25$ & $1-128 \mathrm{nT}$ & 64 \\
& $I_{z}$ & $0.1-500$ & $10^{-14}-10^{-10}$ & 1000 \\
& & & $\mathrm{~A} / \mathrm{cm}^{2} / \sqrt{\mathrm{Hz}}$ & \\
\hline
\end{tabular}


Key parameters are distributed to all INTERBALL participants.

3. Preparation of ASPI data bank on optical disks and/or digital tapes and distribution to all member groups of the ASPI team.

\section{First results}

ASPI instruments OPERA and MIF-M were switched on after the booms were deployed at 12:59 UT on 3 August 1995. The INTERBALL-1 spacecraft was at this time in the magnetosheath just outside the magnetopause on the outbound part of its first orbit. The information was dumped via the analogue telemetry system. On 5 August 1995 the PRAM and OPERA instruments started digital data transmission. On 6 August 1995 the FGM-I magnetometer was switched on. In this section we present initial results of the ASPI observations.

In the following, in order to differentiate between the similar notations of the $X, Y, Z$ components in the spacecraft and GSE frames of reference, GSE components are implied only if explicitly stated. The GSE frame of reference is used only for the DC magnetic and electric field data in the discussions of particular crossings. In all other cases (while discussing sensor performance or speaking about $\mathrm{AC}$ waveforms and spectra) the spacecraft frame of reference is implied.

\subsection{Performance of ASPI sensors}

We describe the background signal levels and sensor noise levels. We also perform a comparison of the INTERBALL-1 data with that from the previous missions at the rather well-studied plasma boundary the bow shock. The detailed analysis of the measurement methods and the spacecraft interferences will be given in the future papers.

\subsubsection{Magnetic field measurements}

In Fig. 2 examples of the magnetic field noise spectra in the quiet solar wind plasma are presented (bottom curves). Curve 5 shows the noise level as measured via the MIF-M filter bank. The search-coil (last four filters, see Sect. 2.3) noise signal $0.02-0.03 \mathrm{pT} / \sqrt{H}_{z}$ is close to the noises of the GEOTAIL and ISEE-1,3 instruments at about $1 \mathrm{kHz}$ (Matsumoto et al., 1994). Curve 3 in Fig. 2 represents the noise level of the waveform signal, measured by the MIF-M flux-gate sensor. The noise level of the flux-gate sensor of the FGM-I magnetometer is similar to that of the MIF-M and is $5 \mathrm{pT} / \sqrt{H}_{z}$.

We also present in Fig. 2 bow shock spectra measured by the MIF-M (curve 2: FFT of the waveform and curve 4: filter bank) and by PROGNOZ-10 BUD-1M experiments (curve 1) (Petrukovich et al., 1993). Similar amplitudes at 1 and $15 \mathrm{~Hz}$ in both spectra prove that

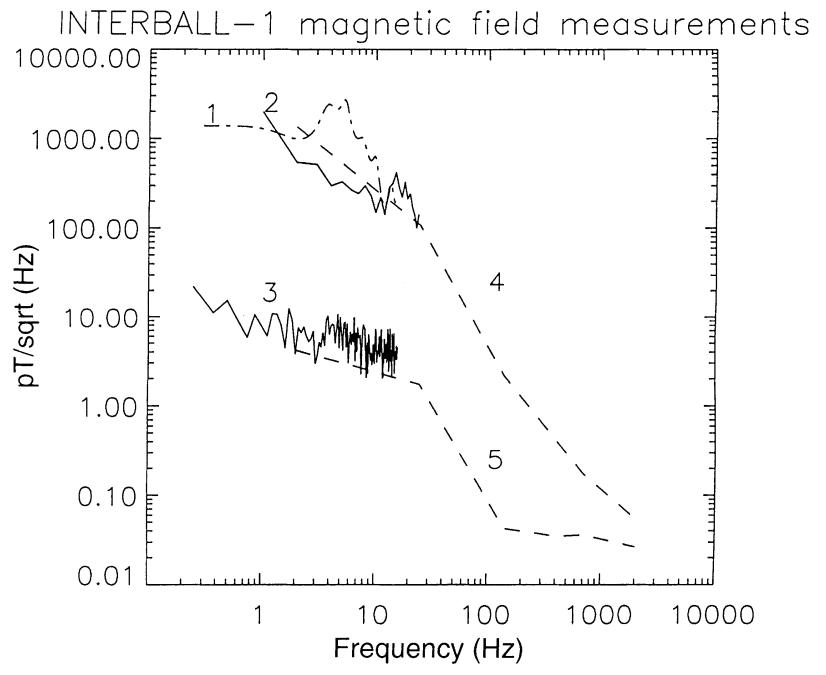

Fig. 2. Noise levels of the magnetic sensors. Curve 1: bow shock spectrum from the PROGNOZ-10 BUD-1M experiment; curve 2: bow shock spectrum (FFT of the wave form); curve 3: noise spectrum (FFT of the waveform); curve 4: bow shock spectrum (filter bank); curve 5: noise spectrum (filter bank)

observations from the two missions correspond to one another. The presence of the spectral peak at a few $\mathrm{Hz}$ is the typical feature of the near-shock turbulence in this frequency range. Its position in the spectrum depends upon local shock and solar wind parameters and can vary in a rather broad frequency range $(4 \mathrm{~Hz}$ in the PROGNOZ-10 spectrum and $20 \mathrm{~Hz}$ in the INTERBALL one).

As for the DC magnetic field measurements, noise levels of both MIF-M and FGM-I flux-gates are to an order of magnitude lower than their quantization levels. The difference between the FGM-I and MIF-M DC magnetic field spectra is about $10-20 \mathrm{pT} / \sqrt{H} z$ except for the frequencies of the harmonics of the 10-s interference signal (to be discussed). In the next subsections with experimental data description, the magnetic field measurements by the MIF-M flux-gate sensor will be used unless explicitly stated otherwise.

The main static magnetic moments of the INTERBALL spacecraft were determined in the EMC chamber of the Lavochkin centre. The on-board measured offsets coincide with the offsets determined on the ground. They are less than $10 \mathrm{nT}$ and stable. By the use of the dual magnetometer method (flux-gate sensors of MIF$\mathrm{M}$ and FGM-I) we can separate the spacecraft interferences from real magnetic field data with an accuracy of 1 $\mathrm{nT}$ in the frequency band $0-0.1 \mathrm{~Hz}$ and of $0.01 \mathrm{nT}$ in 0.1-32 Hz. The most difficult DC magnetic field measurement problem is the offset determination of the $B_{x}$ (non-rotating) component. This offset was determined with an accuracy of $0.3 \mathrm{nT}$ (quantization level of the DC magnetic field signal).

Three major types of magnetic field spacecraft interferences were detected:

1. Quasi-sinusoidal magnetic field signal with a period of about $10 \mathrm{~s}$ and 7-8 $\mathrm{nT}$ amplitude at the position of 
the FGM-I flux-gate sensor (Fig. 1). It is practically undetectable at the position of the MIF-M flux-gate sensor on the same boom. Due to the rather stable phase, this interference can be easily extracted from the data with the use of FFT filtering.

2. Spurious magnetic field bursts radiated by the spacecraft heating system in the range 1-2000 Hz. These impulses have a characteristic appearance in the filterbank data.

3. Quasi-periodic interference with a frequency of about $80 \mathrm{~Hz}$ at the search-coil sensor location. Its amplitude reaches about $10 \%$ of the natural magnetic field waveform amplitudes.

\subsubsection{Electric field measurements}

In Fig. 3 we present the noise levels and characteristic signals of the electric field measurements. Curves 3 and 6 correspond to the background electric field spectra measured by the OPERA instrument in the solar wind. Curve 6 represents the filter-bank spectrum of the signal from the BD1-BD2 dipole $(22 \mathrm{~m})$. The numbers are close to that of the PROGNOZ-8 17-m dipole noise (Aleksevich et al., 1983). Curve 3 represents the OPERA on-board fast Fourier transform (FFT) spectrum summed over three components. Comparing these two curves, one should take into account the less sensitive quantization level of the FFT and a possible overestimate of the electric field by the shorter length dipoles, spectra of which are summed in the FFT output.

Curve 1 in Fig. 3 shows the on-board FFT spectrum detected at the quasi-perpendicular bow shock on 7

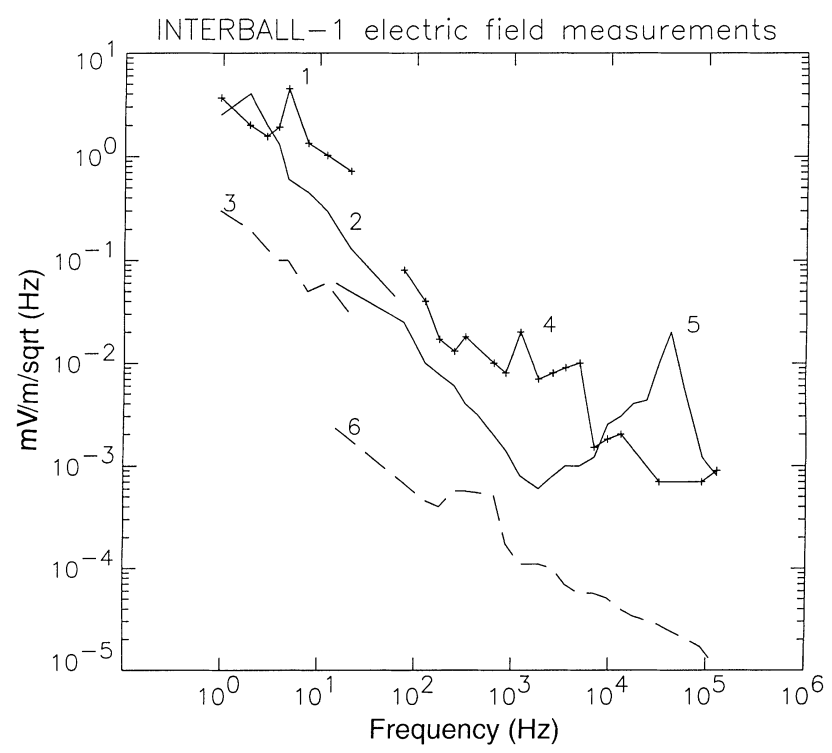

Fig. 3. Noise levels of the electric field sensors. Curve 1: bow shock spectrum (on board FFT); curve 2: bow shock spectrum, measured by the BUD-E experiment of PROGNOZ-8; curve 3: noise spectrum (on board FFT); curve 4: bow shock spectrum (filter bank); curve 5: upstream spectrum (filter bank); curve 6: noise spectrum (filter bank)
August 1995 at 08:34 UT. Comparison with the PROGNOZ-8 bow shock spectrum [curve 2 in Fig. 3 (Nozdrachev et al., 1995)] proves that as in the case of the magnetic field spectra, ASPI ELF measurements are compatible with the results of previous electric field experiments. The OPERA filter-bank spectrum at the same shock is shown as curve 4 (Fig. 3). It is interfered with by one of the spacecraft transmitters at $40-60 \mathrm{kHz}$ (this peak is extracted in the Figure), which reduces the electric field amplitude due to the saturation of the filterbank preamplifier. The plateau at the frequences $0.2-4$ $\mathrm{kHz}$ is characteristic of the ion-acoustic waves at the quasi-perpendicular shocks.

An example of the upstream electric field spectra from the long dipole (curve 5) exhibits a well-defined peak at the Langmuir frequencies. Such peaks are often registered in the electron foreshock of quasi-perpendicular shocks. Its amplitude $(0.02 \mathrm{mV} / \mathrm{m} / \sqrt{H} z)$ is much stronger than the average one, measured by the ISEE-1 (Etcheto and Faucheux, 1984) and IMP-6 (Filbert and Kellogg, 1979) long antennas, but is close to the typical short-dipole measurements on Phobos-1,2 (Trotignon et al., 1991). Taking into account typical wavelengths of Langmuir waves (of order of the Debye length), we believe that the 22-m-long dipole antenna on INTERBALL-1 is more appropriate for studies of highfrequency electrostatic waves.

The preliminary DC OPERA electric field data analysis shows that:

1. In the plasmasphere the DC electric field signals are dominated by the V-cross-B induction signal. The auroral electric fields can be determined by substraction of the model V-cross-B signal.

2. Despite the overall symmetry of long dipole with respect to the spacecraft spin axis, the light nonsymmetry of the boom elements near the BD1 and $\mathrm{BD} 2$ electric probes causes the spin-modulated photocurrent interference signal in the long dipole $\left(E_{y}\right)$ component. This influence is usually less than the natural electric fields in the magnetosheath and dense plasmasheet, but dominates in the solar wind and empty lobes.

3. The short BD3-BD4 dipole ( $E_{z}$ component) is the most symmetric with respect to the spacecraft spin axis, nevertheless the spin-modulated signal induced by photo-current dominates in the DC $E_{z}$ channel.

4. The DC channel of the another short, nearly sunward directed dipole $\left(E_{x}\right.$, probes $\left.\mathrm{BD} 5, \mathrm{BD} 6\right)$ is usually in saturation outside the plasmasphere, introducing difficulties in the measurements of the sunward DC electric field by the short asymmetric dipole. In the dilute plasma once per revolution, photoelectrons from the BD6 probe flow to the boom, making a negative pulse of about $250 \mathrm{mV}$ in the DC $E_{x}$ electric field channel. However, this signal often becomes negligible in the plasmasheet and magnetosheath when the boom is shielded by the plasma with smaller Debye length.

The subtraction of the spacecraft- and photocurrentinduced interferences from the electric field measure- 
ments requires a knowledge of the plasma and magnetic field characteristics, measured by other INTERBALL-1 experiments. Not all these data were available at the time of writing. For this reason in Sect. 3.3 we present only the long-dipole antenna measurements in the magnetosheath and near-Earth plasma sheet. In this particular set of data taken in a sufficiently dense plasma, interferences are proved to be significantly smaller than natural signals. The GSE frame of reference $E_{y}$ component is reconstructed using the spacecraft rotation with no additional processing. The use of shorter-dipole measurements requires the removal of interference signals and will be performed in the future publications.

As for the AC electric field waveforms, presented in Sect. 3.3, we also consider long-dipole measurements as the most accurate. The signal is given in its original form as recorded on board. Signals from the shorter diploes are modified with the preliminary calibration factors relative to the long-dipole signal.

\subsubsection{Plasma current measurements}

In Fig. 4 curves 2 and 3 show the FGM-I split Langmuir probe signal $\left(I_{z}\right)$ spectrum from five MIF-M filters. Curve 3 represents a typical quiet solar wind signal, whose amplitudes are several times less than the sensitivity threshold of the PROGNOZ-10 split probe at $25 \mathrm{~Hz}$ (Romanov et al., 1991). Before the ASPI experiment, current fluctuation measurements had never been performed in the outer magnetosphere in the frequency range $130-2000 \mathrm{~Hz}$.

Unfortunately, during first two months of the INTERBALL-1 operation no bow-shock crossings were

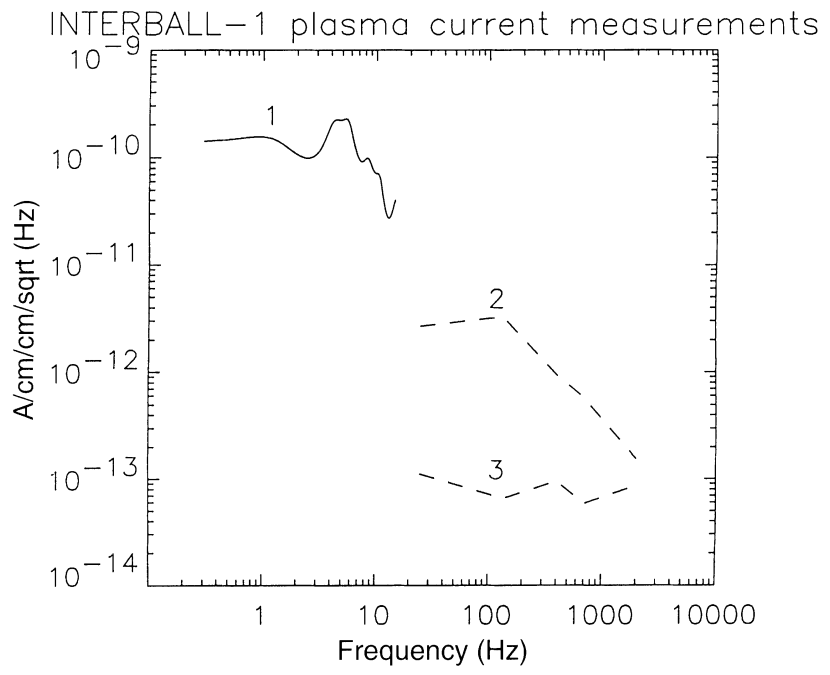

Fig. 4. Noise level of the split Langmuir probe. Curve 1: plasma current spectrum at the bow shock, detected by the PROGNOZ-10 plasma wave experiment; curve 2: bow shock plasma current spectrum from the filter bank; curve 3: split probe noise as measured by the filter bank in the quiet solar wind registered during transmission of the $I_{z}$ high-resolution waveform signal with a $500-\mathrm{Hz}$ sampling rate. The plasma current spectrum presented in Fig. 4 (curve 2) was measured by the MIF-M filter bank at the lowlatitude shock crossing on 6 August 1995. PROGNOZ10 [Fig. 4, curve 1, (Petrukovich et al., 1993)] and INTERBALL spectra agree well, taking into account power-law extrapolation to the adjacent frequency range.

The main $I_{z}$ waveform interference was found to be the same as for the search-coil wide-band channel, namely - a quasi-periodic signal at frequency about $80 \mathrm{~Hz}$. This interference can partially saturate the channel in the regions with the high level of wave turbulence (e.g. bow shock or outer cusp).

\subsection{High-latitude magnetopause measurements}

We start our experimental data presentation with an example of the high-latitude magnetospheric boundary crossing. The INTERBALL-1 crossed the outbound flank magnetopause on 26 August at 04:39:22 UT, at the point with the GSE coordinates $\left(-2.3,-9.0,9.1 \mathrm{R}_{E}\right)$. In Fig. 5 the DC magnetic field data is presented in the magnetospheric boundary coordinate system (Russell and Elphic, 1978). The magnetosphere region is on the left. The normal direction was found as a vector product of the average magnetic fields on both sides of the discontinuity. The magnetic field rotation and the drop of its total value $B_{t}$ occur at 04:39:22 UT. Before

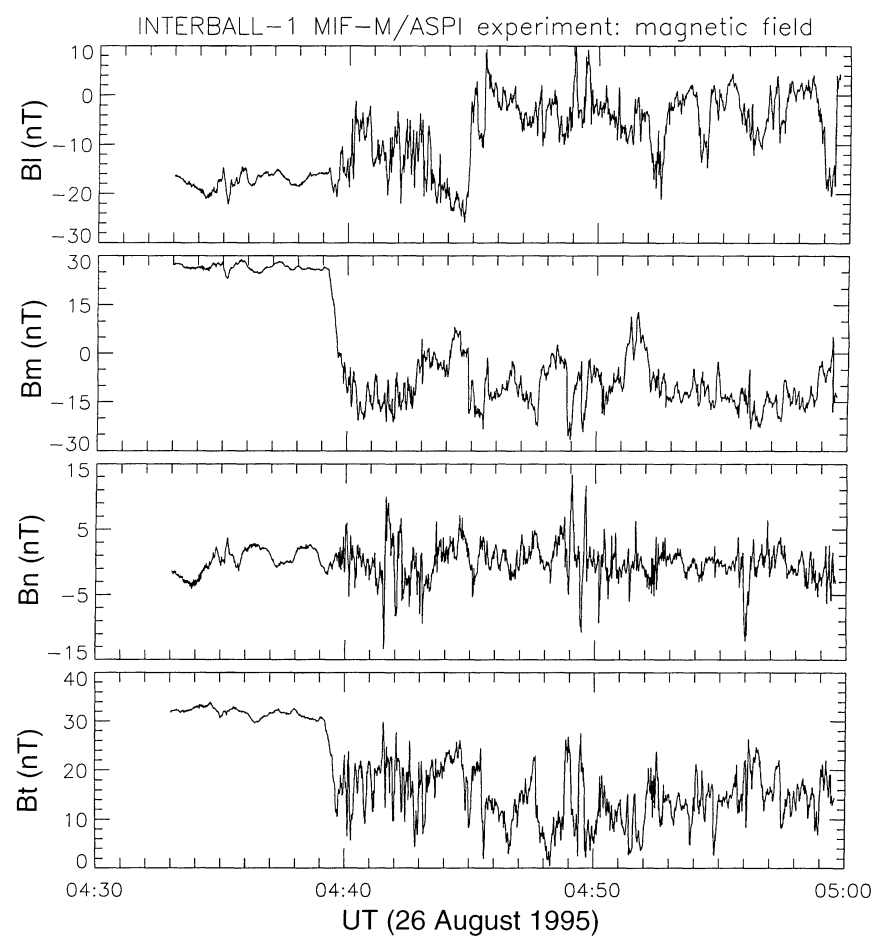

Fig. 5. High-latitude magnetopause crossing 26 August 1995. Magnetic field components $B_{l}, B_{m}, B_{n}$ and total value $B_{t}$ in the boundary frame of reference. See text for details 
this moment a wave with the period of about $130 \mathrm{~s}$ is seen in the $B_{n}$ component, being absent in the $B_{t}$. We believe that this signature is due to the magnetopause surface wave propagation. Just outside of the magnetopause at 04:40:10 UT one can see a wave packet in the $B_{n}$ component, coinciding with the rotation of the $B_{l}$ one.

The 150-s-long time-interval starting at 04:38:10 UT was chosen for the minimum variance data analysis, the result is presented in Fig. 6. The FGM-I magnetic field data is used for this short time-interval analysis as the FGM-I instrument had a sampling rate four times higher than that of the MIF-M in this particular case. The ratios of the maximum-to-intermediate and intermediate-to-minimum eigenvalues are 38 and 5, respectively. The minimum variance direction is just a few degrees apart from the normal direction. Also, the eigenvector frame of reference is rotated about $10^{\circ}$ around the normal with respect to the boundary frame used in Fig. 5. The bipolar disturbances in the $B_{k}$ (Fig. 6) and $B_{n}$ (Fig. 5) components are similar to the flux transfer event signatures (Russell and Elphic, 1978), which were investigated for first time at the high latitudes by PROGNOZ-8 (Blecki et al., 1987, 1988; Savin, 1994). A comparison with the MHD simulations of the magnetopause (Nikutowski et al., 1996) was performed. It demonstrates the similarity of this event with either the magnetosheath pressure-pulse dynamics or with the Kelvin-Helmholtz instability development. Both models predict significant fluctuations of the normal magnetic field in the second half of the crossing. No similarity with the crossings of the quiet magnetopause or reconnection region was found. However, the latter should be checked more carefully using the threedimensional local reconnection model.

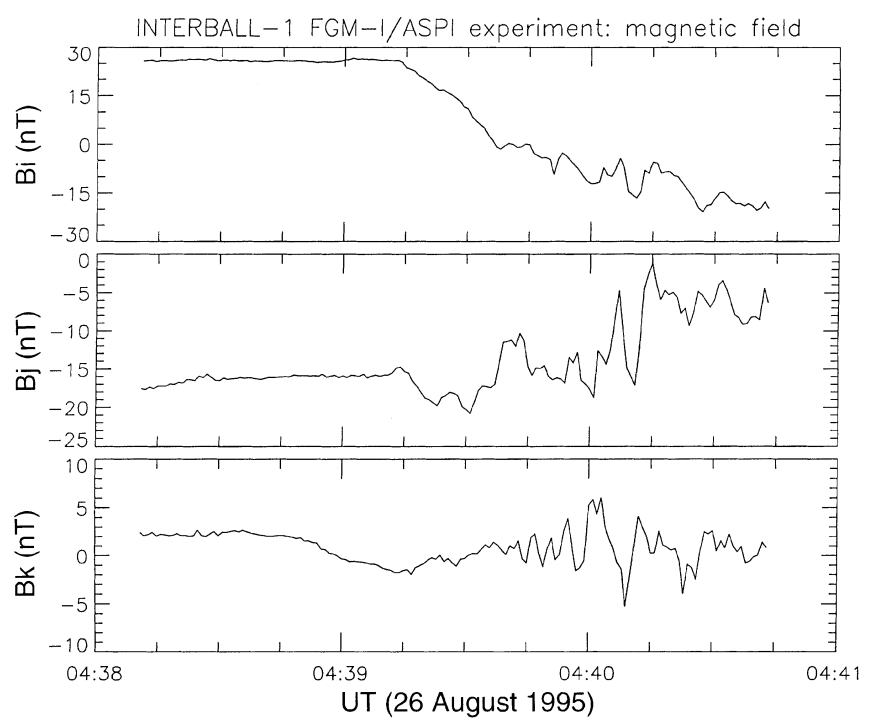

Fig. 6. High-latitude magnetopause crossing 26 August 1995. Magnetic field components in the minimum variance frame of reference. $B_{i}$ : maximum variance direction; $B_{j}$ : intermediate variance direction; $B_{k}$ : minimum variance direction. See text for details
In Fig. 6 one can point out low-frequency waves with a frequency lower than $0.1 \mathrm{~Hz}$ and with $0.2-0.3-\mathrm{Hz}$ frequencies (close to the ion gyro-frequency). At high latitudes the second type of near-magnetopause wave was classified and studied in the PROGNOZ-8,10 data (Vaisberg et al., 1983; Klimov et al., 1986; Belova et al., 1991; Savin 1994). The higher-frequency ELF wave activity can be studied with the help of Fig. 7 where FFT spectrograms of MIF-M and OPERA waveforms are displayed. The upper panel shows $0.1-32 \mathrm{~Hz}$ electric field $E_{z}$ component spectra, the bottom panel shows the same for the $B_{x}$ component. The behaviour of the other components is similar to that of presented ones. The magnetosheath encounter is seen on the magnetic spectrogram at 04:39:22 UT as the beginning of the intense signal. Such a signal is known to be a good indicator of the magnestosheath plasma. However, the electric field spectra do not exhibit critical magnetosphere/magnetosheath differences in the amplitudes. Outside the magnetosphere they correlate well with the intense bursts of magnetic spectra. The electric field bursts at the inner border of magnetopause at 04:39 UT and at 04:35 UT are almost electrostatic, with up to 8 $\mathrm{mV} / \mathrm{m}$ peak-to-peak amplitudes. To our knowledge, we are the first to show the presence of a weak magnetic component during such bursts, which themselves are rather usual for the high-latitude magnetopause crossings (Vaisberg et al., 1983; Klimov, 1992). Magnetic spectra also have a wider frequency spread in comparison with the electric ones, as the magnetic field channel is substantially more sensitive.

Just outside the magnetopause at 04:39-04:46 UT there exists a region with very high turbulence level (Fig. 7), in which the amplitude of the electric field signal is more than $5 \mathrm{mV} / \mathrm{m}$, and that of the magnetic field more than $5 \mathrm{nT}$. In this region the $B_{l}$ component (Fig. 5) is highly disturbed but does not vanish to the nearly zero average level, as happens deeper in the magnetosheath. So, this component here is closer to its value inside the magnetosphere. This feature could be due to the effect of the shocked solar wind magnetic field lines draping around the magnetosphere. We will henceforth call this region the "draped layer" (DL). In the DL the biggest ELF wave bursts correlate with the bipolar $B_{n}$ signatures in Fig. 5. Outside DL the turbulence amplitudes are weaker, bursts are rarer and at 05:28 UT (not shown on the plots) the turbulence characteristics become finally typical for the quiet magnetosheath with amplitudes several times less than in the DL. We call the regions with the turbulence features as at 04:39-05:00 UT the "turbulent zones" (TZ).

The high-latitude $\mathrm{TZ}$ was reported first based on the PROGNOZ-10 data and then studied in detail using the PROGNOZ-8 crossings (Savin et al., 1990; Belova et al., 1991; Savin , 1994; Klimov, 1992). The studies of DL at the flank magnetopause were unfortunately not performed in the first reports on such observations by PROGNOZ-8,10 spacecraft (Klimov et al., 1986; Sokolov et al., 1990; Savin, 1994). In the INTERBALL-1 flank magnetopause crossings studied so far (18 Octo- 


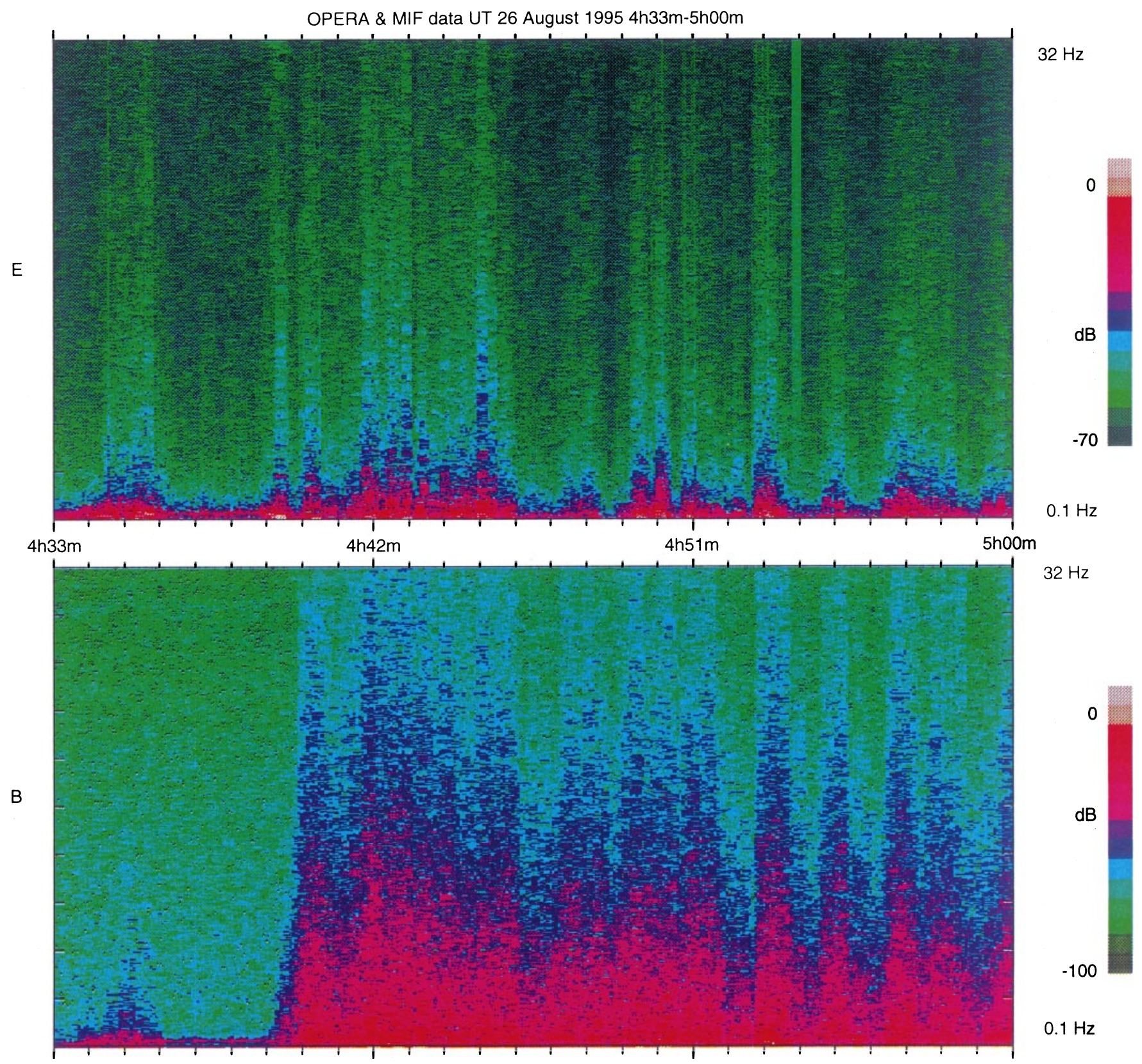

Fig. 7. High-latitude magnetopause crossing 26 August 1995. Spectrograms of the $E_{x}$ (top) and $B_{z}$ (bottom) waveform signals. Frequency range is $0.1-32 \mathrm{~Hz}$

ber, 18-20 UT; 2 September, 19:00 UT; 10 September, 13:22 UT; 30 August, 00-01 UT) TZ and DL regions are always present except for the 30 August crossing, where the DL could not be identified due to a very complex multiple magnetopause encounter.

So, the described flank outbound magnetopause crossing is a fairly representative one. It is compatible with the results of earlier missions. As in the case of the PROGNOZ-8 crossing (Blecki et al., 1987, 1988; Savin, 1994), in future analysis we expect to find the accelerated electrons and ions in the magnetopause vicinity and especially in the "turbulent zone".

\subsection{Observations at low latitudes}

In August/September 1995 at the inbound part of orbits, INTERBALL-1 was crossing the low-latitude magnetopause, the low-latitude boundary layer and the plasmasheet. The case of the 21 September 1995 crossing was chosen for the presentation. Here the plasma sheet, predicted by the orbital analysis (Prokhorenko, 1995) was observed together with the unexpected magnetopause crossing.

In Fig. 8 the ASPI GSE magnetic field data for the interval 02:00-03:00 UT, 21 September 1995 are shown. 


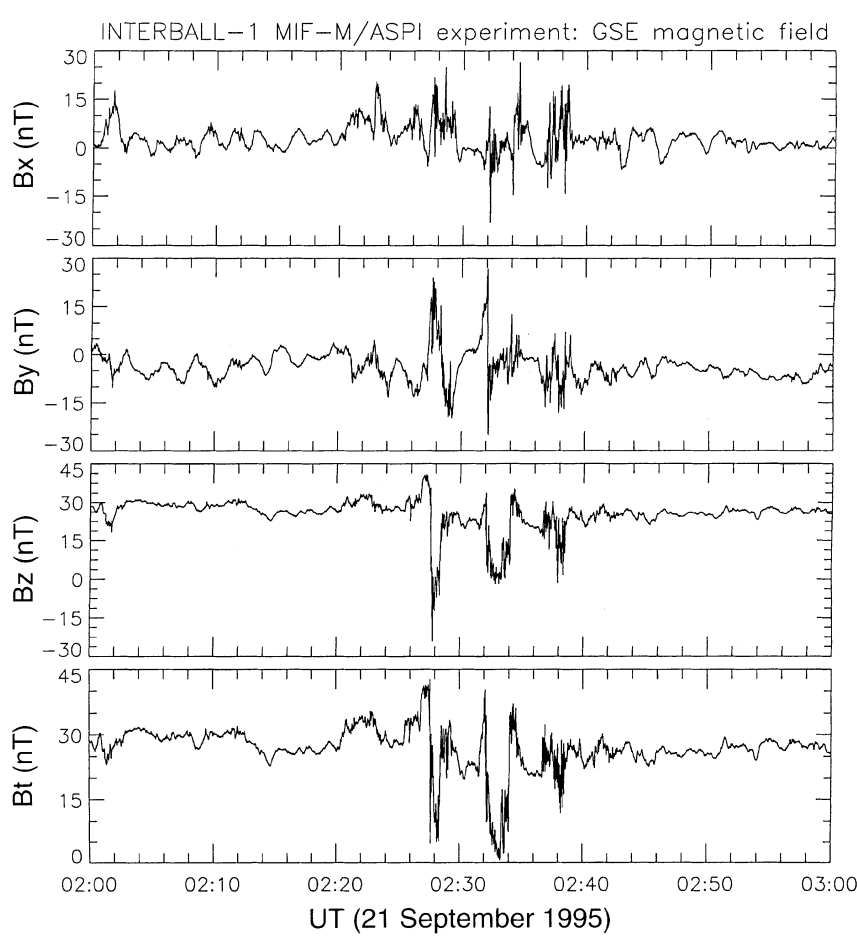

Fig. 8. Low-latitude boundary crossing 21 September 1995. GSE frame-of-reference magnetic field components and total value

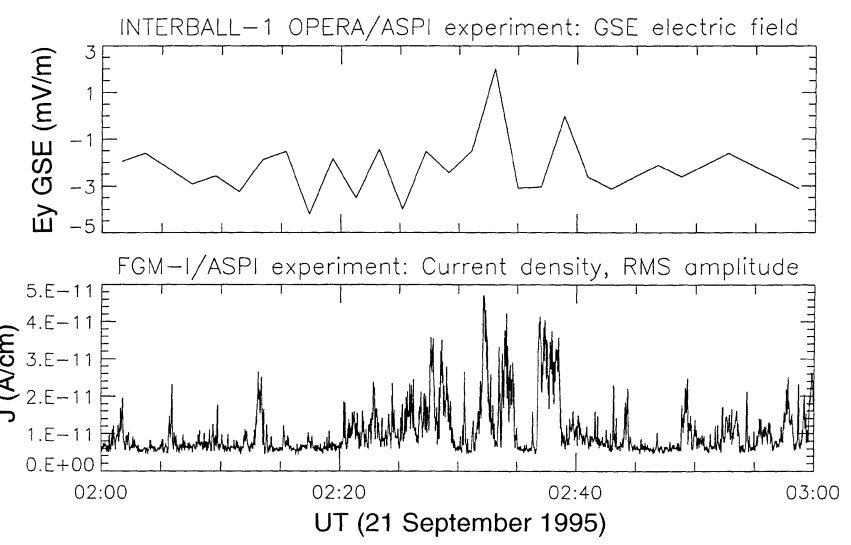

Fig. 9. Low-latitude boundary crossing 21 September 1995. GSE frame-of-reference $E_{y}$ electric field component (top panel) and RMS amplitude of the plasma current fluctuations (bottom panel)

On the top of Fig. 9 the GSE frame-of-reference $E_{y}$ component, measured by the long dipole of the electric field instrument is shown. Only for this dipole the interference signal is proved to be negligible in this particular crossing (see Sect. 3.1.2). As the spacecraft spin period is about $120 \mathrm{~s}$, the resulting electric field values are available only once per $60 \mathrm{~s}$. On the bottom of Fig. 9 the RMS amplitude of the plasma current oscillations in the range $1-8 \mathrm{~Hz}$ is plotted. FFT spectrograms for the same period are in Fig. 10.

The GSE spacecraft coordinates for the middle of the interval are $\left(-3.4,-12,-1.8 \mathrm{R}_{E}\right)$. In the GSM frame only the $Z$ GSM coordinate differs substantially from the
GSE counterpart, being equal to $2 \mathrm{R}_{E}$. The Tsyganenko model for $k_{p}=2$ predicts GSE magnetic field vector at 02:00 UT to be $\left(5.3,8.5,15.7 \mathrm{nT}, B_{t}=18.6 \mathrm{nT}\right)$. One can conclude that excluding the disturbed interval at 02:26-02:39 UT the experimentally observed magnetosphere is slightly compressed, having an average total magnetic field value 1.4 times higher than the model one and a nearly vanishing $B_{y}$ GSE component.

In the middle of Fig. 8 at $02: 28$ and 02:33 UT $B_{t}$ drops to 3-10 nT, which means most probably that the spacecraft is out of the magnetosphere. Preliminary analysis of the plasma particle data confirms this conclusion. At the 02:38 UT only the near-magnetopause turbulent layer is touched for about $2 \mathrm{~min}$. Similar to the high-latitude magnetopause observations the magnetosheath and magnetopause turbulent layer encounters are accompanied by intense electromagnetic noise (Figs. 9, 10). The most intense wave bursts correlate with sharp magnetic field changes.

The exit from the magnetosheath at the 02:33 UT is the only one long enough to determine the value of the DC electric field from the spin-modulated signal of the long dipole. The $E_{y}$ GSE component here is about 2.5 $\mathrm{mV} / \mathrm{m}$ (Fig. 9). It corresponds to anti-solar plasma velocity of the order of $350 \mathrm{~km} / \mathrm{s}$, which is reasonable for the magnetosheath. In the plasma sheet the $E_{y}$ GSE component is negative with an average value of -2.5 $\mathrm{mV} / \mathrm{m}$, which implies sunward plasma convection with a speed of about $100 \mathrm{~km} / \mathrm{s}$. Such convection takes place during the northward interplanetary magnetic field, and exactly this IMF direction was registered during this period.

The interval 02:32:00-02:32:25 UT, with the most pronounced magnetopause crossing, was chosen for the detailed analysis of the AC waveform data (Fig. 11). The magnetopause crossing occurs at 02:32:06-02:32:08 UT. The upper panel of Fig. 11 shows the magnetic field waveform signal. Three AC electric field waveforms are on the bottom panels. The highest-amplitude (up to 10 $\mathrm{nT}$ !) $B_{z}$ wave packet is seen exactly at the time interval of the DC-field sharp rotation (02:32:08 UT). The frequency of the dominating signal is about $0.8-1.5 \mathrm{~Hz}$. The most intense electric field signal with amplitudes greater than $5 \mathrm{mV} / \mathrm{m}$ is seen just inside the magnetopause.

Electrostatic waves with a frequency of a few $\mathrm{Hz}$ and amplitude of about $10 \mathrm{mV} / \mathrm{m}$ just inside the highlatitude dayside magnetopause were reported for the first time using one-component measurements by Vaisberg et al. (1983). They were later described for the flank magnetopause crossings by the PROGNOZ-8,10 spacecraft (Klimov et al., 1986; Blecki et al., 1988; Belova et al., 1991; Savin et al., 1990; Klimov, 1992; Savin, 1994), and for the low-latitude magnetopause by ISEE-1 and GEOTAIL (Cattell et al., 1995) spacecraft. In Fig. 11 vector measurements of such waves are presented for the first time.

The higher-frequency waves can be studied in Fig. 10. The $E_{x}$ spectra at the magnetic disturbances have an intense $1-10-\mathrm{Hz}$ component (red tones), while in the rest of the magnetosphere the $E_{x}$ waveform quan- 


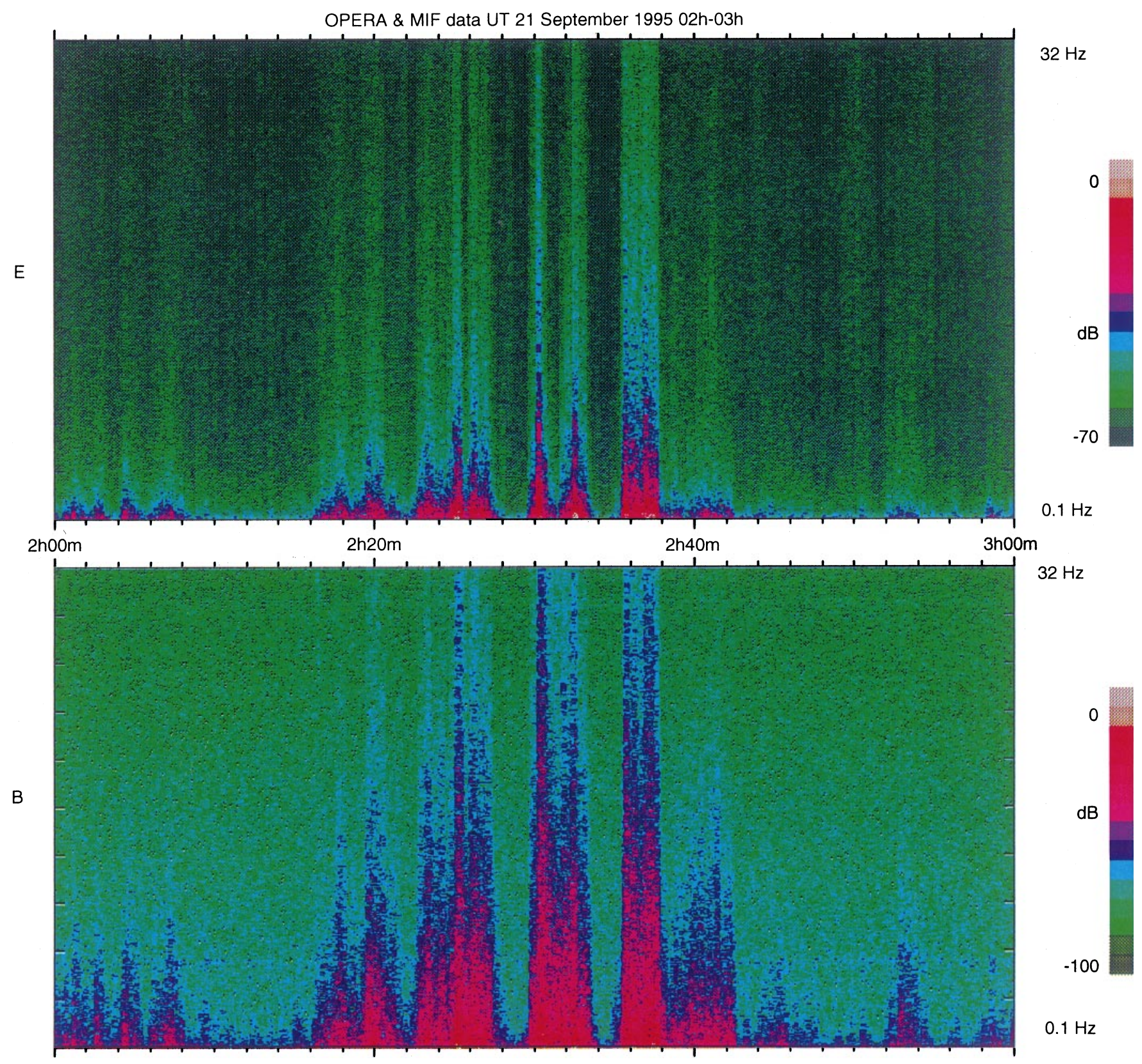

Fig. 10. Low-latitude magnetopause crossing 21 September 1995. Spectrograms of the $E_{x}$ (top) and $B_{z}$ (bottom) waveform signals. Frequency range is $0.1-32 \mathrm{~Hz}$

tization is too low. The magnetic field channel, in contrast, registers the $1-10-\mathrm{Hz}$ waves outside such disturbances. The comparison with the plasma current $1-8-\mathrm{Hz}$ fluctuations (bottom panel of Fig. 9) shows that at $02: 20-02: 45$ UT the spikes in the plasma current signal are similar to that of the $B_{z}$ spectra (Fig. 10). At 02:06, 02:09, 02:14, 02:49, 02:56-03:00 UT there are intense events in Fig. 9, which have no counterparts in the dynamic spectra. We suppose that at these moments electrostatic waves with smaller amplitudes and higher frequencies were observed. As the split probe signal has better quantization than the electric field one, such emissions were registered only by the split probe.
Turning now to the plasma sheet region (Fig. 8, and 10 excluding 02:26-02:39 UT) we would like to point out the electromagnetic waves in the $0.1-20-\mathrm{Hz}$ frequency range. Such waves were found to be characteristic for the plasma sheet [see e.g. Blecki et al., (1994) and references therein]. However, most of the previous results were based only on the electric field data studies. At 02:0502:12 UT the modulation of the magnetic spectra can be seen. The DC magnetic field components at this time also fluctuate with a period of $1.5-2.5 \mathrm{~min}$. The absence of such oscillations in the plot of the total magnetic field value could speak in favour of either an Alfvén wave or a magnetopause surface wave observation. 


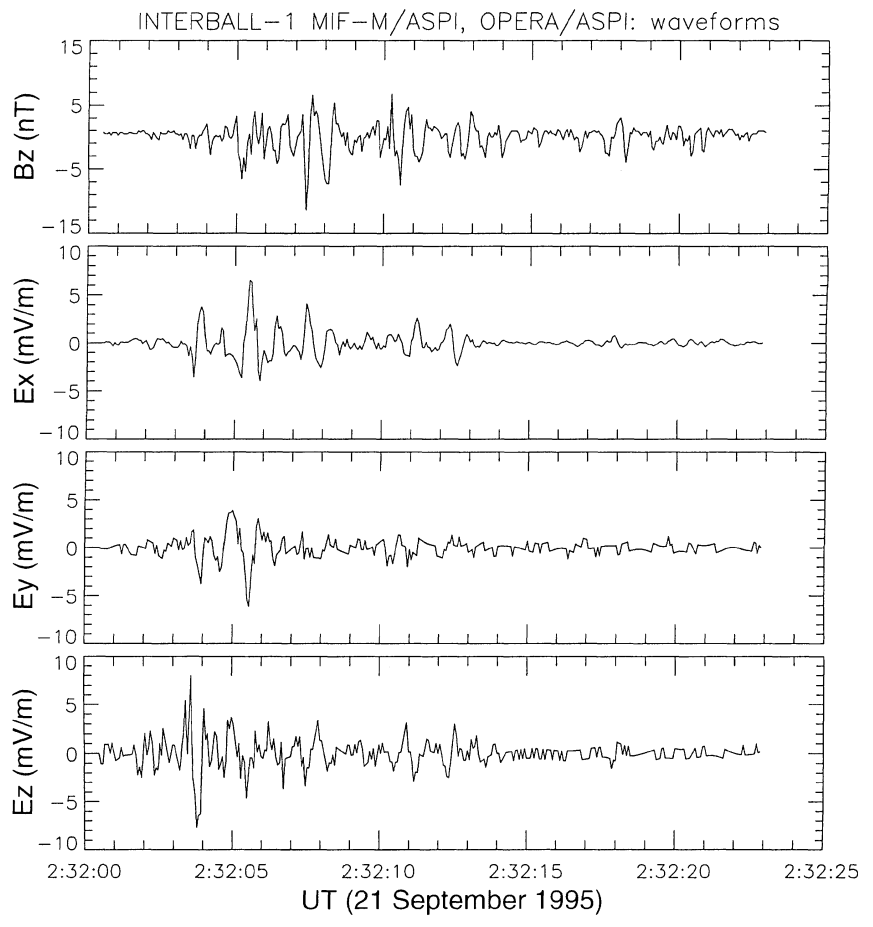

Fig. 11. Low-latitude boundary crossing 21 September 1995. Waveform snapshots. From top to bottom: $B_{z}$ magnetic field: $E_{x}, E_{y}, E_{z}$ electric field waveform components in the spacecraft frame of reference (see text for details)

An example of the low-latitude magnetospheric boundary crossing described here is rather unusual for such a spacecraft position. It demonstrates a substantial ELF wave activity both in the relatively quiet and in the highly disturbed periods. Special interest is attracted by the electric and magnetic field oscillations near the magnetopause transition, which have frequencies near 1 $\mathrm{Hz}$. Their amplitudes can reach $10 \mathrm{nT}$ and $5 \mathrm{mV} / \mathrm{m}$, respectively. We have also confirmed the existence of the ELF electric oscillations in the plasma sheet and measured their magnetic component. The described crossing can be subjected to in-depth studies on a multiinstrument basis, as nearly all INTERBALL-1 experiments were operating in fast modes.

\section{Conclusions}

The ASPI wave and field experiment on board the INTERBALL-1 spacecraft has been successfully operating in the orbit beginning from the 3 August 1995. Unique features of the ASPI instruments, such as reliable multi-sensor magnetic field measurements, three-component electric field measurements and electric current measurements make it possible to provide valuable new information on the plasma dynamics in the magnetosphere.

Analysis of the in-flight instrument operations have shown the high quality and reliability of the DC magnetic field measurements. The MIF-M/PRAM DC magnetometer is working as the primary magnetic field experiment of the mission. AC magnetic field measurements are performed at high sensitivity in the few- $\mathrm{Hz}$ range, thus covering the gap usually existing in the frequency range between the DC and AC magnetic field experiments. Measurements of the electric field and plasma current have sufficient sensitivity to study a variety of plasma processes. However, three-component DC electric field measurements have encountered difficulties which are mainly due to the non-symmetry of the short booms with respect to the Sun and the spacecraft. Preliminary analysis of the data collected shows a compatibility of new results with those of previous missions.

Several examples of data registered in the various regions of the Earth's magnetosphere are described in the second half of the paper. The high-latitude magnetopause crossing is a typical representative of such phenomena. A number of crossings similar to the presented one were encountered by INTERBALL-1 during the first months of operation. Being combined with the recent advances in theory and numerics these data collections will permit a thorough investigation of inherent plasma processes forming the magnetospheric boundaries.

An example of the low-latitude magnetospheric boundary crossing described is rather unusual for such a spacecraft position. It demonstrates substantial ELF wave activity both in the relatively quiet and in the highly disturbed periods. Properties of these waves correspond well to the results of the previous experiments and of the high-latitude INTERBALL-1 ones. We have confirmed the existence of intense electric fluctuations in the few-Hz frequency range and have studied their relation to simultaneously observed intense magnetic waves. The described crossing can be subjected to in-depth studies on a multi-instrument basis, as nearly all INTERBALL-1 experiments were operating in fast modes.

In all described cases high-resolution waveform measurements provide a good basis for the studies of the ELF turbulence.

The unique constellation of magnetospheric spacecraft planned to be in orbit in the immediate future (GEOTAIL, INTERBALL-1,2, WIND, POLAR) is another promising perspective to perform global studies of the Earth's magnetosphere.

Acknowledgements. The ASPI team is grateful to F. Scarf and C. Russell for their useful ideas during ASPI design. We wish to thank A. Galeev and L. Zelenyi for their help in data interpretation. We also thank other INTERBALL experimenters G. Zastenker, N. Borodkova, E. Budnik, A. Fedorov, Yu. Yermolaev for preliminary particle data. The ASPI team also greatly appreciates the participation in the project preparation and operation of the Lavochkin association specialists G. Sukhanov, I. Tserenin, A. Nevzorov, A. Rusanov, S. Gorkin, V. Nikiforov, I. Sobachkin, V. Larin, V. Nagornykh, A. Grigorjev, F. Dolgopolicheskij and of the Space Research Institute specialists L. Pesotskij, R. Melnikov, 
S. Shamaev, V. Nazarov, T. Lesina, Yu. Agafonov, L. Belyakova, M. Yanovskij, Yu. Lissakov, V. Prokhorenko, M. Mogilevskij, E. Gavrilova.

Topical Editor K. -H. Glaßmeier thanks a referee for his help in evaluating this paper.

\section{References}

Aleksevich, Ya. N., Yu. V. Afanasiev, V. M. Balebanov, V. E. Baskakov, Yu. N. Bobkov, N. F. Borodin, O. L. Vaisberg, Yu. Voita, V. N. Gorobey, V. N. Karachevsky, S. I. Klimov, V. E. Korepanov, N. I. Nazarov, S. G. Namestnik, K. Novak, V. Novakevich, M. N. Nozdrachev, C. Oberz, A. M. Pevzner, L. V. Pesotsky, V. N. Porfirov, S. P. Savin, Z. Sikorsky, P. P. Timofeev, P. Triska, and B. N. Turchaninov, Complex wave experiment aboard satellite PROGNOZ-8 (in Russian), Kosm. Issled., 21, 4418, 1983.

Belova, E. V., J. Blecki, M. Denis, L. M. Zeleny, and S. P. Savin, Excitation of ion cyclotron waves at the boundary of the magnetosphere, Sov. J. Plasma Phys., 17, 555, 1991.

Bering, E. A., M. C. Kelley, and F. S. Mozer, Split Langmuir probe measurement of current density and electric fields in an aurora, J. Geophys., Res.,78, 2201, 1973a.

Bering, E. A., M. C. Kelley, F. S. Mozer, and U. V. Fahleson, Theory and operation of the split Langmuir probe, Planet. Space Sci., 21, 1983, 1973b.

Blecki, J., K. Kossacki, S. I. Klimov, M. N. Nozdrachev, A. N. Omelchenko, S. P. Savin, and A. Yu. Sokolov, ELF/ULF plasma waves observed on PROGNOZ-8 near the magnetopause, Artif. Satell. 22, 5, 1987.

Blecki, J., K. Kossacki, B. Popielawska, S. I. Klimov, S. A. Romanov, S. P. Savin, and L. M. Zelenyi, ELF plasma waves associated with plasma jets near the Earth magnetopause as observed by PROGNOZ-8, Phys. Scr., 37, 623, 1988.

Blecki, J., B. Nikutowski, P. Koperski, B. Popielawska, K. Kossacki, S. A. Romanov, and S. P. Savin, ELF plasma waves in the hot and cold plasma fluxes observed by PROGNOZ-8 in the magnetospheric tail, Ann. Geophysicae, 12, 1006, 1994.

Cattell, C. F, J. Wygant, F. S. Moser, T. Okada, K. Tsuruda, S. Kokubun, and T. Yamamoto, ISEE-1 and GEOTAIL observations of low-frequency waves at the magnetosphere, J. Geophys. Res., A100, 11823, 1995.

Etcheto, J., and M. Faucheux, Detailed study of electron plasma waves upstream of the Earth's bow shock, J. Geophys. Res., A89, 6631, 1984.

Fahleson, U., Theory of electric field measurements conducted in the magnetosphere with electric probe, Space Sci. Rev., 7, 338, 1967.

Filbert, P. C., and P. J. Kellogg, Electrostatic noise at the plasma frequency beyond the Earth's bow shock, J. Geophys. Res., A84, 1369, 1979.

Grard, R., C. Beghin, M. Mogilevsky, Yu. Mikhailov, O. Molchanov, A. Pedersen, J. -G. Trotignon, and V. Formisano, Electric field and plasma observations near Halley's Comet (in Russian) Pisma A. Zh., 12, 683, 1986.

Grard R., D. Klinge, S. Klimov, S. Savin, and J. G. Trotignon, The plasma wave system on Phobos, J. Phys. E., 22, 656, 1989.

Gurnett, D. A., Electric field and plasma observations in the magnetosphere, in Theoretical problems of magnetospheric physics, Ed. IUCSTP secretariat. Washington, p. 123, 1972.

Klimov, S. I., Energetics of ULF/ELF plasma waves in the solar wind and outer Earth's magnetosphere, ESA SP-346, 95, 1992.

Klimov, S. I., M. N. Nozdrachev, P. Triska, Ya. Voita, A. A. Galeev, Ya. N. Aleksevich, Yu. V. Afanasiev, V. E. Baskakov, Yu. N. Bobkov, R. B. Dunetz, A. M. Zhdanov, V. E. Korepanov, S. A. Romanov, S. P. Savin, A. Yu. Sokolov, and V. S. Shmelev, Investigation of plasma waves by combined wave diagnostic device BUDWAR (PROGNOZ-10-INTERCOSMOS) (in Russian), Kosm. Issled., 24, 177, 1986.

Klimov, S. I., Z. Kravchek, V. E. Korepanov, S. P. Savin, V. M. Balebanov, G. Simonenko, C. Novak, and Ya. Aleksevich, Plasma waves investigation aboard VEGA spacecraft, (in Russian), Kosm. Issled., 25, 943, 1987.

Klimov, S., S. Romanov, E. Amata, J. Blecki, J. Büchner, J. Juchniewicz, J. Rustenbach, P. Triska, L. J. C. Woolliscroft, S. Savin, Yu. Afanas'yev, U. de Angelis, U. Auster, G. Bellucci, A. Best, F. Farnik, V. Formisano, P. Gough, R. Grard, V. Grushin, G. Haerendel, V. Ivchenko, V. Korepanov, H. Lehmann, B. Nikutowski, M. Nozdrachev, S. Orsini, M. Parrot, A. Petrukovich, J. L. Rauch, K. Sauer, A. Skalsky, J. Slominski, J.G. Trotignon, J. Vojta, and R. Wronowski, ASPI experiment: Measurements of fields and waves onboard of the INTERBALL-TAIL mission, INTERBALL mission and payload, IKICNES, p. 120, 1995.

Matsumoto, H., I. Nagano, R. R. Anderson, H. Kojima, K. Hashimoto, M. Tsutsui, T. Okada, I. Kimura, Y. Omura, and M. Okada, Plasma wave observations with GEOTAIL, spacecract, J. Geomagn Geoelectr., 46, 59, 1994.

Mozer, F. S., Comparison of spherical double probe electric field measurements with plasma bulk, Geophys. Res. Lett., 10, 737, 1983.

Nikutowski, B., J. Büchner, G. Haerendel, L. Lemke, J. Rustenbach, H. Wiechen, A. Otto, R. Walker, S. Klimov, and S. Savin, Magnetopause normal determination with the INTERBALL measurements, Abst. AGU 1995 Fall Meeting, F512, 1996.

Nozdrachev, M. N., A. A. Petrukovich, and J. Juchniewicz, ULF/ ELF monochromatic oscillations observed by PROGNOZ-8 and -10 spacecraft during quasi-perpendicular supercritical shock crossings, Ann. Geophysicae., 13, 573, 1995.

Nozdrachev, M. N., V. A. Styazhkin, A. A. Zarutsky, S. I. Klimov, S. P. Savin, A. A. Skalsky, A. A. Petrukovich, Yu. V. Lissakov, I. S. Arshinkov, N. Abadgiev, and A. Bochev, Magnetic field measurements onboard the INTERBALL TAIL spacecraft: INTERBALL mission and payload, the FM-3I instrument, IKI-CNES, p. 228, 1995.

Petrukovich, A. A., S. A. Romanov, and S. I. Klimov, Dispersion characteristics of plasma emissions near the quasi-perpendicular Earth's bow shock observed by PROGNOZ-10 spacecraft, Proc. START conf. ESA WPP-047, Ed. C. P. Escoubet, ESA Publications division, Noordwijk, The Netherlands. p. $281,1993$.

Pikelner, S. B., and V. N. Tsytovich, Field annihilation and particle acceleration in solar flares (in Russian) Astron. Zh., 52, 738, 1975.

Prokhorenko, V. I., Mission analysis for the INTERBALL project. Pre-launch orbit selection and long-term experiments planning, INTERBALL mission and payload, IKI-CNES, p. 46, 1995.

Romanov, S. A., S. I. Klimov, and P. A. Mironenko, Experimental derivation of ELF waves dispersion relations and evidence of wave coupling in the Earth's bow shock foot from the data of the PROGNOZ-10, Adv. Space Res., 11, 19, 1991.

Russell, C. T., and R. C. Elphic, Initial ISEE magnetometer results: magnetopause observations, Space Sci. Rev., 22, 681, 1978.

Savin, S. P., ELF waves near the high-latitude magnetopause, Abstr. AGU Chapman conf. Phys. Magnetosph., March 14-18, Ed. published by American Geophysical Union. No other information available. p. 41, 1994

Savin, S. P., L. M. Zelenyi, and V. I. Prokhorenko, Specific tasks of multiple-scale plasma measurements in Regatta-Cluster system, Proc. Intern. Worksh. Space Plasma Phys. Investig. by Cluster and Regatta, Graz, Austria, Ed. 1990: Ed. E. J. Rolfe, ESA Publications division, Noordwijk. The Netherlands. ESA SP306, p. 7, 1990.

Sokolov, A. Yu., I. K. Khabibrakhmanov, M. M. Kuznetsova, J. Lemaire, M. Roth, J. M. Lesceux, and F. Verheest, Detection of 
nonlinear features in plasma and field measurements by PROGNOZ-8, ESA SP-311, 81, 1990.

Trotignon, J. G., M. Hamelin, R. Grard, A. Pedersen, S. Klimov, S. Savin, A. Skalsky, and C. Kennel, A comparison between the Earth's and Mars' bow shocks detected by the PHOBOS plasma-wave system, Planet. Space Sci., 39, 99, 1991.

Tunally, J. K. E., The effect of noise on probe measurements in the magnetosphere, Ann. Geophys., 26, 853, 1970.
Vaisberg, O. L., A. A. Galeev, L. M. Zelenyi, G. N. Zastenker, A. N. Omelchenko, S. I. Klimov, S. P. Savin, Yu. I. Yermolaev, V. N. Smirnov, and M. N. Nozdrachev, Fine structure of the magnetopause from the measurements of the satellites PROGNOZ-7 and PROGNOZ-8 (in Russian), Kosm. Issled., 21, 57, 1983.

Vaisberg, O. L., S. I. Klimov, and V. E. Korepanov, Current density measurements near the bow shock by the split Langmuir probe (in Russian), Kosm. Issled., 27, 461, 1989. 